\title{
DIFFUSION APPROXIMATIONS FOR THE MAXIMUM OF A PERTURBED RANDOM WALK
}

\author{
VICTOR F. ARAMAN, ${ }^{*}$ New York University \\ PETER W. GLYNN, ${ }^{* *}$ Stanford University
}

\begin{abstract}
Consider a random walk $S=\left(S_{n}: n \geq 0\right)$ that is 'perturbed' by a stationary sequence $\left(\xi_{n}: n \geq 0\right)$ to produce the process $S=\left(S_{n}+\xi_{n}: n \geq 0\right)$. In this paper, we are concerned with developing limit theorems and approximations for the distribution of $M_{n}=\max \left\{S_{k}+\xi_{k}: 0 \leq k \leq n\right\}$ when the random walk has a drift close to 0 . Such maxima are of interest in several modeling contexts, including operations management and insurance risk theory. The associated limits combine features of both conventional diffusion approximations for random walks and extreme-value limit theory.
\end{abstract}

Keywords: Perturbed random walk; diffusion approximation; light-tailed distribution; heavy-tailed distribution; limit theorem

2000 Mathematics Subject Classification: Primary 60F17; 60J60; 60G99

Secondary 60G70; 90B30

\section{Introduction}

Let $S=\left(S_{n}: n \geq 0\right)$ be a random walk sequence such that $S_{0}=0$ and $S_{n}=X_{1}+\cdots+X_{n}$, where the $X_{i}$ are independent and identically distributed. Given a sequence $\left(\xi_{n}: n \geq 0\right)$ of 'perturbations', we call the process $\left(S_{n}+\xi_{n}: n \geq 0\right)$ a 'perturbed random walk'. In this paper, we are concerned with developing limit theorems and related approximations for the maximum

$$
M_{n}=\max _{0 \leq k \leq n}\left(S_{k}+\xi_{k}\right) .
$$

'Perturbed random walks' have previously been studied in the insurance risk theory literature; see, for example, Gerber (1970), Schmidli (1995), and Schlegel (1998). In contrast with the perturbations studied in those papers, which have generally been themselves of random walk type, we shall assume that $\left(\xi_{n}: n \geq 0\right)$ is a stationary sequence. This stationarity assumption also means that our perturbed random walks cannot be viewed through the prism of nonlinear renewal theory (see, for example, Woodroofe (1982)), because our perturbations typically violate the 'uniform continuity in probability' hypothesis that is common in that literature. The class of stationary perturbation discussed here is both natural from a modeling standpoint, and reflects a middle ground between the perturbations of random walk type (in which the perturbations are of the same magnitude as the unperturbed random walk) and those arising in nonlinear renewal theory (in which the perturbations are, in a sense, asymptotically constant;

Received 5 February 2004; revision received 1 March 2005.

* Postal address: Stern School of Business, New York University, New York, NY 10012-1126, USA.

Email address: varaman@stern.nyu.edu

** Postal address: Management Science and Engineering, Stanford University, Stanford, CA 94305-5015, USA.

Email address: glynn@stanford.edu 
see Glasserman and Liu (1997) for a particular case of such perturbations appearing in an inventory production context).

For our class of perturbed random walk, the large-time behavior of $S_{n}+\xi_{n}$ is largely explained by that of the random walk $S$ itself. On the other hand, the maximum $M_{n}$ inherits some of the extreme-value behavior of the perturbations. Consequently, the limit theory that appears in this paper combines features of the classical diffusion approximations for conventional random walks with certain characteristics associated with the extreme-value behavior of $\left(\xi_{n}: n \geq 0\right)$.

As indicated above, the distribution of $M_{n}$ is relevant to a variety of applications. In particular,

1. $M_{n}$ has the distribution of the time spent by order $n$ in a make-to-order production facility, in which possible delays in the delivery of supplier components are explicitly modeled;

2. $M_{n}$ arises as the end-to-end delay for the $n$th data packet in a communications network with multiple paths connecting the source node to the destination node; and

3. $\mathrm{P}\left(M_{\infty}>x\right)$ is the ruin probability for an insurer having initial reserve $x$, when certain customers do not necessarily pay their premiums on time.

We refer the reader to Araman and Glynn (2004) for further details of these modeling applications. Araman and Glynn (2004) also studied the tail probability $\mathrm{P}\left(M_{\infty}>x\right)$ as $x \rightarrow \infty$ and developed a number of different asymptotics for both heavy-tailed and light-tailed perturbations.

In contrast, our interest here is on studying approximations for $M_{n}$ when the random walk $S$ has drift close to 0 . This setting is of great importance, in view of our modeling applications. In particular, asserting that $S$ is almost driftless lets us model, for example, a make-to-order facility that is running at close to $100 \%$ utilization. In addition, in the insurance risk setting, by making this assumption we can model a marketplace in which the insurer can set its premiums at a rate only slightly higher than the average payout rate. Of course, from a mathematical standpoint, this nearly driftless setting corresponds to the environment in which diffusion approximations for (conventional) random walks are applicable.

We present four different types of result. In Section 2, we discuss finite-time diffusion approximations for $M_{n}$ when the perturbations are light tailed, whereas, in Section 3, we describe the corresponding theory for heavy-tailed perturbations. In Section 4, we provide a diffusion approximation for $M_{\infty}$ in the light-tailed case and, in Section 5, we conclude the paper with a description of approximations for $M_{\infty}$ in the presence of heavy tails.

\section{Finite-horizon limit theory with light tails}

To rigorously describe such heavy-traffic limit theory, we must consider a family of perturbed random walks that is parameterized by the mean of the increment random variable. To this end, consider a perturbed random walk that describes a processing facility with 'balanced loading', so that the increment random variables $\left(X_{i}: i \geq 1\right)$ have mean 0 . For $\mu \in \mathbb{R}$, let $X_{i}(\mu)=X_{i}+\mu$ and let $S_{n}(\mu)=\sum_{i=1}^{n} X_{i}(\mu)$ for $n \geq 0$. Furthermore, we shall permit the distribution of the perturbation to depend upon $\mu$. Specifically, for the perturbed random walk indexed by $\mu$, let $\xi_{i}(\mu)=\kappa(\mu) \xi_{i}$ for some appropriately chosen $\kappa(\cdot)$. We shall be concerned with the behavior of the maximum random variable

$$
M_{n}(\mu)=\max _{0 \leq k \leq n}\left(S_{k}(\mu)+\xi_{k}(\mu)\right)
$$

for $\mu$ close to 0 . 
The assumption that we impose on the $X_{i}$ (when they describe a system with balanced loading), namely that $\left(S_{n}: n \geq 0\right)$ satisfy a functional central limit theorem or invariance principle, is standard in the literature on heavy-traffic limit theory. By ' $\stackrel{\mathrm{D}}{=}$ ' we denote equality in distribution.

Assumption 1. Suppose that there exist constants $\sigma$ and $p \geq 2$ and a probability space supporting both a sequence of random variables $\left(X_{i}^{\prime}: i \geq 1\right)$ and a standard Brownian motion $B=(B(t): t \geq 0)$ for which

(i) $\left(X_{i}^{\prime}: i \geq 1\right) \stackrel{\mathrm{D}}{=}\left(X_{i}: i \geq 1\right)$ and

(ii) $\sum_{i=1}^{n} X_{i}^{\prime}=\sigma B(n)+o\left(n^{1 / p}\right)$ almost surely (a.s.) as $n \rightarrow \infty$.

The precise form of Assumption 1 is that of a 'strong approximation' hypothesis. Such an assumption is valid when the $X_{i}$ are independent and identically distributed with $\mathrm{E}\left|X_{i}\right|^{p}<\infty$ for $p>2$; see p. 107 of Csörgő and Révész (1981). However, this hypothesis is also known to be valid for a large class of dependent $X_{i}$; see, for example, Philipp and Stout (1975) and Csáki and Csörgő (1995). Since the $X_{i}^{\prime}$ appearing in Assumption 1 share the same joint distributions as those of the $X_{i}$, we can and will henceforth assume that the probability space supporting the $X_{i}$ is that guaranteed by Assumption 1 .

We use the following notation: by ' $\stackrel{\mathrm{D}}{\rightarrow}$ ' we denote convergence in distribution, by $\lfloor\cdot\rfloor$ we denote the greatest-integer function, and by $\lceil\cdot\rceil$ we denote the least-integer function. Now consider our assumptions on the perturbations.

Assumption 2. Suppose that ( $\left.\xi_{j}: j \geq 0\right)$ is a stationary sequence for which there exist positive constants $\gamma$ and $\beta$ such that

$$
\frac{\max _{0 \leq k \leq n} \xi_{k}}{(\log n)^{\gamma}} \stackrel{\mathrm{D}}{\rightarrow} \beta
$$

as $n \rightarrow \infty$.

Assumption 2 holds, for example, when the $\xi_{j}$ are independent and identically distributed with a right tail satisfying

$$
\frac{\log \mathrm{P}\left(\xi_{j}>x\right)}{x^{1 / \gamma}} \rightarrow-\beta^{-1 / \gamma}
$$

as $x \rightarrow \infty$. However, Assumption 2 continues to be valid under quite modest dependency hypotheses on the $\xi_{j}$; see Glynn and Zeevi (2000) for details. It should be noted that the tail condition (1) holds for $\xi_{j}$ that have Gaussian, gamma, or Weibull (right) tails.

We are now ready to state our first heavy-traffic limit theorem.

Theorem 1. Assume that Assumption 1 and Assumption 2 hold, and suppose that $\kappa(\mu) \sim$ $c\left(|\mu|(\log (1 /|\mu|))^{\gamma}\right)^{-1}$ as $\mu$ either decreases or increases to 0 , where $c$ is a positive constant. Then

$$
|\mu| M_{\left\lfloor t / \mu^{2}\right\rfloor}(\mu) \stackrel{\mathrm{D}}{\rightarrow} \max _{0 \leq s \leq t}(\sigma B(s)-s)+2^{\gamma} \beta c
$$

as $\mu \nearrow 0$, and

$$
|\mu| M_{\left\lfloor t / \mu^{2}\right\rfloor}(\mu) \stackrel{\mathrm{D}}{\rightarrow} \max _{0 \leq s \leq t}(\sigma B(s)+s)+2^{\gamma} \beta c
$$

as $\mu \searrow 0$. 
Proof. We consider only the case in which $\mu \nearrow 0$, so that $\mu$ is negative throughout the proof; the case $\mu \searrow 0$ can be handled analogously. We start by noting that the upper bound

$$
|\mu| M_{\left\lfloor t / \mu^{2}\right\rfloor}(\mu) \leq|\mu| \max _{0 \leq k \leq\left\lfloor t / \mu^{2}\right\rfloor} S_{k}(\mu)+|\mu| \max _{0 \leq k \leq\left\lfloor t / \mu^{2}\right\rfloor} \xi_{k}(\mu)
$$

clearly holds. For the lower bound, fix an $\varepsilon>0$ and use the path-by-path uniform continuity of Brownian motion to choose an $l$ large enough that

$$
\mathrm{P}\left(\max _{\substack{0 \leq|s-u| \leq t / l \\ 0 \leq s, u \leq t}}|(\sigma B(s)-s)-(\sigma B(u)-u)|>\frac{1}{3} \varepsilon\right)<\varepsilon .
$$

Let $k^{*}$ be a maximizer of $\max \left\{S_{k}(\mu): 0 \leq k \leq\left\lfloor t / \mu^{2}\right\rfloor\right\}$, let $t(i)=i t / l$, and let $i^{*}=\left\lfloor l \mu^{2} k^{*} / t\right\rfloor$, meaning that $t\left(i^{*}\right) \leq \mu^{2} k^{*} \leq t\left(i^{*}+1\right)$. We then have the following lower bound:

$$
\begin{aligned}
|\mu| M_{\left\lfloor t / \mu^{2}\right\rfloor}(\mu) \geq|\mu| \max _{t\left(i^{*}\right) \leq \mu^{2} k \leq t\left(i^{*}+1\right)}\left(S_{k}(\mu)+\xi_{k}(\mu)\right) \\
\geq|\mu| \min _{t\left(i^{*}\right) \leq \mu^{2} k \leq t\left(i^{*}+1\right)} S_{k}(\mu)+|\mu| \max _{t\left(i^{*}\right) \leq \mu^{2} k \leq t\left(i^{*}+1\right)} \xi_{k}(\mu) \\
\geq|\mu| \max _{0 \leq k \leq\left\lfloor t / \mu^{2}\right\rfloor} S_{k}(\mu)-|\mu| \max _{\substack{\mu^{2}|k-j| \leq t / l \\
0 \leq k, j \leq\left\lfloor t / \mu^{2}\right\rfloor}}\left|S_{k}(\mu)-S_{j}(\mu)\right| \\
+|\mu| \min _{0 \leq i<l} \max _{i t / l \mu^{2} \leq k<(i+1) t / l \mu^{2}} \xi_{k}(\mu) .
\end{aligned}
$$

It follows from the upper bound (2) and the lower bound (3) that

$$
\begin{aligned}
& || \mu\left|M_{\left\lfloor t / \mu^{2}\right\rfloor}(\mu)-\right| \mu\left|\max _{0 \leq k \leq\left\lfloor t / \mu^{2}\right\rfloor} S_{k}(\mu)-2^{\gamma} \beta c\right| \\
& \leq|| \mu\left|\max _{0 \leq k \leq\left\lfloor t / \mu^{2}\right\rfloor} \xi_{k}(\mu)-2^{\gamma} \beta c\right| \\
& \quad+|| \mu\left|\min _{\substack{0 \leq i<l \\
i t / l \mu^{2} \leq k<(i+1) t / l \mu^{2}}} \xi_{k}(\mu)-2^{\gamma} \beta c\right| \\
& \quad+|\mu| \max _{\substack{\mu^{2}|k-j| \leq t / l \\
0 \leq k, j \leq\left\lfloor t / \mu^{2}\right\rfloor}}\left|S_{k}(\mu)-S_{j}(\mu)\right| .
\end{aligned}
$$

On the basis of Assumption 1, standard arguments imply that

$$
|\mu| \max _{0 \leq k \leq\left\lfloor t / \mu^{2}\right\rfloor} S_{k}(\mu) \stackrel{\mathrm{D}}{\rightarrow} \max _{0 \leq s \leq t}(\sigma B(s)-s)
$$

and

$$
|\mu| \max _{\substack{\mu^{2}|k-j| \leq t / l \\ 0 \leq k, j \leq\left\lfloor t / \mu^{2}\right\rfloor}}\left|S_{k}(\mu)-S_{j}(\mu)\right| \stackrel{\mathrm{D}}{\rightarrow} \max _{\substack{0 \leq|s-u| \leq t / l \\ 0 \leq s, u \leq t}}|(\sigma B(s)-s)-(\sigma B(u)-u)|
$$

as $\mu \nearrow 0$; see Glynn (1998), for example. Also, Assumption 2 shows that

$$
|\mu| \max _{0 \leq k \leq\left\lfloor t / \mu^{2}\right\rfloor} \xi_{k}(\mu)=|\mu| \kappa(\mu) \max _{0 \leq k \leq\left\lfloor t / \mu^{2}\right\rfloor} \xi_{k} \stackrel{\mathrm{D}}{\rightarrow} 2^{\gamma} \beta c
$$


as $\mu \nearrow 0$. The stationarity of the $\xi_{j}$ then implies that

$$
|\mu| \max _{i t / l \mu^{2} \leq k<(i+1) t / l \mu^{2}} \xi_{k}(\mu) \stackrel{\mathrm{D}}{\rightarrow} 2^{\gamma} \beta c
$$

as $\mu \nearrow 0$, from which it follows easily that

$$
|\mu| \min _{0 \leq i<l} \max _{i t / l \mu^{2} \leq k<(i+1) t / l \mu^{2}} \xi_{k}(\mu) \stackrel{\mathrm{D}}{\rightarrow} 2^{\gamma} \beta c
$$

as $\mu \nearrow 0$. Consequently, (4)-(8) establish that

$$
\begin{aligned}
& \mathrm{P}\left(|| \mu\left|M_{\left\lfloor t / \mu^{2}\right\rfloor}(\mu)-\right| \mu\left|\max _{0 \leq k \leq\left\lfloor t / \mu^{2}\right\rfloor} S_{k}(\mu)-2^{\gamma} \beta c\right|>\varepsilon\right) \\
& \leq \mathrm{P}\left(|| \mu\left|\max _{0 \leq k \leq\left\lfloor t / \mu^{2}\right\rfloor} \xi_{k}(\mu)-2^{\gamma} \beta c\right|>\frac{\varepsilon}{3}\right) \\
& +\mathrm{P}\left(|| \mu\left|\min _{0 \leq i<l} \max _{i t / l \mu^{2} \leq k<(i+1) t / l \mu^{2}} \xi_{k}(\mu)-2^{\gamma} \beta c\right|>\frac{\varepsilon}{3}\right) \\
& +\mathrm{P}\left(|\mu| \max _{\substack{\mu^{2}|k-j| \leq t / l \\
0 \leq k, j \leq\left\lfloor t / \mu^{2}\right\rfloor}}\left|S_{k}(\mu)-S_{j}(\mu)\right|>\frac{\varepsilon}{3}\right) \\
& \rightarrow \mathrm{P}\left(\max _{\substack{0 \leq|s-u| \leq t / l \\
0 \leq s, u \leq t}}|(\sigma B(s)-s)-(\sigma B(u)-u)|>\frac{\varepsilon}{3}\right) \\
& <\varepsilon
\end{aligned}
$$

as $\mu \nearrow 0$. Thus, we have proved that

$$
|| \mu\left|M_{\left\lfloor t / \mu^{2}\right\rfloor}(\mu)-\right| \mu\left|\max _{0 \leq k \leq\left\lfloor t / \mu^{2}\right\rfloor} S_{k}(\mu)-2^{\gamma} \beta c\right| \stackrel{\mathrm{D}}{\rightarrow} 0
$$

as $\mu \nearrow 0$. A 'converging together' argument, together with (5), proves the theorem.

We note that $\max _{0 \leq s \leq t}(\sigma B(s)-s) \stackrel{\mathrm{D}}{=} X(t)$, where $X=(X(t): t \geq 0)$ is a reflecting Brownian motion with drift -1 and variance parameter $\sigma^{2}$, conditional on starting from the origin. As a consequence, the limiting random variable appearing in Theorem 1 is known to have a closed-form distribution; see, for example, Harrison (1985).

The magnitude of $\kappa(\mu)$ is chosen in Theorem 1 such that we obtain a limiting regime in which both the random walk $\left(S_{n}: n \geq 0\right)$ and the perturbation $\left(\xi_{j}: j \geq 0\right)$ influence the limiting random variable. (If $\kappa(\mu)$ is chosen to go to infinity more slowly than is specified in Theorem 1 , the limit random variable turns out to be the same reflecting Brownian motion as would appear in the standard heavy-traffic limit for the unperturbed random walk $\left(S_{n}: n \geq 0\right)$, whereas if $\kappa(\mu)$ is chosen to go to infinity more rapidly, the limit is governed only by the distribution of the $\xi_{j}$.)

Intuitively, we expect to obtain better approximations to the distribution of the maximum of the perturbed random walk when the approximating limit random variable depends on the distributions of both the underlying random walk and the associated perturbations. For a given perturbed random walk $\left(S_{j}+\xi_{j}: j \geq 0\right)$, the approximation suggested by Theorem 1 is

$$
\max _{0 \leq k \leq n}\left(S_{k}+\xi_{k}\right) \stackrel{\mathrm{D}}{\approx} \max _{0 \leq s \leq n}(\sigma B(s)+\mu s)+(\log n)^{\gamma} \beta,
$$


where ‘ $\stackrel{\text { D }}{\approx}$ ' denotes 'approximate equality in distribution' (and is intended to have no rigorous meaning) and the parameters $\mu, \sigma^{2}, \gamma$, and $\beta$ are obtained from (1) and the relations

$$
\mu=\lim _{n \rightarrow \infty} \frac{1}{n} \mathrm{E} S_{n}
$$

and

$$
\sigma^{2}=\lim _{n \rightarrow \infty} \frac{1}{n} \operatorname{var} S_{n}
$$

We define $\mu$ and $\sigma^{2}$ as in (10) and (11) because Theorem 1 permits the increments of the underlying random walk to be dependent, in which event (10) and (11) are the appropriate relations that typically define $\mu$ and $\sigma^{2}$. It should be further noted that Theorem 1 permits some dependency in the $\xi_{j}$ (see Glynn and Zeevi (2000) for details), and further allows the random walk and the perturbations to be correlated. Permitting such a dependency can be useful. For example, the processing times at the server (in Example 1 of Araman and Glynn (2004), say) may be correlated with the perturbations. Indeed, supplier delay may be a consequence of a large order, which in turn may also cause the processing time for that order at the production facility to be longer than normal.

With regard to the mathematical validity of the approximation (9), Theorem 1 proves that the approximation should be good over spatial scales of order $1 /|\mu|$ when $|\mu|$ is small, the time $n$ is of order $1 / \mu^{2}$, and the perturbations are quite large (of order $\kappa(|\mu|)$ ).

Unlike the conventional heavy-traffic limit theorem for queues, Theorem 1 cannot be derived by directly applying a continuous-mapping argument to a functional limit theorem for the perturbed random walk

$$
\left(|\mu|\left(S_{\left\lfloor t / \mu^{2}\right\rfloor}(\mu)+\xi_{\left\lfloor t / \mu^{2}\right\rfloor}(\mu)\right): t \geq 0\right) .
$$

The reason is that $|\mu| S_{\left\lfloor t / \mu^{2}\right\rfloor}(\mu)$ is converging (weakly) to a process with continuous paths (namely Brownian motion), while, as $\mu \nearrow 0,\left(|\mu| \xi_{\left\lfloor t / \mu^{2}\right\rfloor}(\mu): t \geq 0\right)$ takes both very large and very small values in any time interval of positive length, precluding the possibility that its limit can live on a function space like $D[0, \infty)$, the space of right-continuous functions on $[0, \infty)$ with left limits. As a consequence,

$$
\left(|\mu|\left(S_{\left\lfloor t / \mu^{2}\right\rfloor}(\mu)+\xi_{\left\lfloor t / \mu^{2}\right\rfloor}(\mu)\right): t \geq 0\right)
$$

typically does not obey a functional limit theorem.

Nevertheless, there is a functional analog of Theorem 1. In particular, the techniques used in proving Theorem 1 can be used to show that, as $\mu \nearrow 0$,

$$
|\mu| M_{\left\lfloor\cdot / \mu^{2}\right\rfloor}(\mu) \stackrel{\mathrm{D}}{\rightarrow} X(\cdot)+2^{\gamma} \beta c
$$

in $D[0, \infty)$, where $X=(X(t): t \geq 0)$ is a reflecting Brownian motion with drift -1 and variance parameter $\sigma^{2}$, conditioned on $X(0)=0$; a corresponding limit theorem holds when $\mu \searrow 0$. Note that the presence of the maximum in the defining relation for the $M_{n}$ means that $\left(M_{n}: n \geq 0\right)$ only 'sees' large values of the perturbations, so that the presence of the small values causes no difficulties. Thus, the mathematical issues raised in the previous paragraph do not arise here. 


\section{Finite-horizon limit theory with heavy tails}

We now turn to studying heavy-traffic limit theory when the perturbations have a heavy tail'. In particular, we replace Assumption 2 with the following hypothesis.

Assumption 3. Suppose that $\left(\xi_{j}: j \geq 0\right)$ is a sequence of independent and identically distributed random variables, independent of the sequence $\left(X_{j}: j \geq 1\right)$ and having a common distribution given by

$$
\mathrm{P}\left(\xi_{j}>x\right)= \begin{cases}1, & x \leq b, \\ \left(\frac{b}{x}\right)^{\alpha}, & x \geq b,\end{cases}
$$

for some positive constants $b$ and $\alpha$.

Thus, Assumption 3 is concerned with the special case in which the heavy-tailed perturbations follow a Pareto distribution. Note also that Assumption 3 demands more independence than does Assumption 2.

As in Theorem 1, our heavy-tailed, heavy-traffic limit theorem concerns the parameterized family of perturbed random walks given by

$$
\left(\sum_{j=1}^{n} X_{j}+n \mu+\kappa(\mu) \xi_{n}: n \geq 0\right) .
$$

Theorem 2. Assume that Assumption 1 and Assumption 3 hold, and suppose that $\kappa(\mu)=$ $|\mu|^{(2-\alpha) / \alpha}$. Then

$$
\begin{aligned}
\mathrm{P}\left(|\mu| M_{\left\lfloor t / \mu^{2}\right\rfloor}(\mu)>x\right) & \\
\rightarrow & 1-\operatorname{E} \exp \left(-b^{\alpha} \int_{0}^{t}(x-\sigma B(s)+s)^{-\alpha} \mathrm{d} s\right) \mathbf{1}\left(\max _{0 \leq s \leq t}(\sigma B(s)-s) \leq x\right)
\end{aligned}
$$

as $\mu \nearrow 0$ and

$$
\begin{aligned}
\mathrm{P}\left(|\mu| M_{\left\lfloor t / \mu^{2}\right\rfloor}(\mu)>x\right) & \\
& \rightarrow 1-\operatorname{E} \exp \left(-b^{\alpha} \int_{0}^{t}(x-\sigma B(s)-s)^{-\alpha} \mathrm{d} s\right) \mathbf{1}\left(\max _{0 \leq s \leq t}(\sigma B(s)+s) \leq x\right)
\end{aligned}
$$

as $\mu \searrow 0$.

Proof. As in the proof of Theorem 1, we prove only the case in which $\mu \nearrow 0$. We start by observing that Assumption 1 implies

$$
\begin{aligned}
|\mu| M_{\left\lfloor t / \mu^{2}\right\rfloor}(\mu) & =\max _{0 \leq k \leq\left\lfloor t / \mu^{2}\right\rfloor}\left(|\mu| S_{k}(\mu)+|\mu|^{2 / \alpha} \xi_{k}\right) \\
& =\max _{0 \leq k \leq\left\lfloor t / \mu^{2}\right\rfloor}\left(|\mu|(\sigma B(k)+k \mu)+|\mu|^{2 / \alpha} \xi_{k}\right)+o(1) \quad \text { a.s. }
\end{aligned}
$$

as $\mu \nearrow 0$. However, the scaling properties of Brownian motion yield the distributional equality

$$
\max _{0 \leq k \leq\left\lfloor t / \mu^{2}\right\rfloor}\left(|\mu|(\sigma B(k)+k \mu)+|\mu|^{2 / \alpha} \xi_{k}\right) \stackrel{\mathrm{D}}{=} \max _{0 \leq k \leq\left\lfloor t / \mu^{2}\right\rfloor}\left(\sigma B\left(\mu^{2} k\right)-\mu^{2} k+|\mu|^{2 / \alpha} \xi_{k}\right) .
$$


Furthermore, the independence of the perturbations and the random walk (and, consequently, the Brownian motion) show that

$$
\begin{aligned}
& \mathrm{P}\left(\max _{0 \leq k \leq\left\lfloor t / \mu^{2}\right\rfloor}\left(\sigma B\left(\mu^{2} k\right)-\mu^{2} k+|\mu|^{2 / \alpha} \xi_{k}\right)>x\right) \\
&= \mathrm{P}\left(\max _{0 \leq k \leq\left\lfloor t / \mu^{2}\right\rfloor}\left(\sigma B\left(\mu^{2} k\right)-\mu^{2} k\right)>x-b|\mu|^{2 / \alpha}\right) \\
&+\mathrm{P}\left(\max _{0 \leq k \leq\left\lfloor t / \mu^{2}\right\rfloor}\left(\sigma B\left(\mu^{2} k\right)-\mu^{2} k\right) \leq x-b|\mu|^{2 / \alpha},\right. \\
&= \mathrm{P}\left(\max _{\left.0 \leq k \leq \leq t / \mu^{2}\right\rfloor}\left(\sigma B\left(\mu^{2} k\right)-\mu^{2} k\right)>x-b|\mu|^{2 / \alpha}\right) \\
&+\mathrm{E}\left[1-\prod_{k=0}^{\left\lfloor t / \mu^{2}\right\rfloor}\left(1-\bar{F}_{\xi}\left(\left(x-\sigma B\left(\mu^{2} k\right)-\mu^{2} k+|\mu|^{2 / \alpha} \xi_{k}\right)>x\right)\right.\right. \\
&\left.\times \mathbf{1}\left(\max _{0 \leq k \leq\left\lfloor t / \mu^{2}\right\rfloor}\left(\sigma B\left(\mu^{2} k\right)-\mu^{2} k\right) \leq x-b|\mu|^{2 / \alpha}\right)\right],
\end{aligned}
$$

where $\bar{F}_{\xi}(x)=\mathrm{P}\left(\xi_{j}>x\right)$. The path continuity of Brownian motion shows that

$$
\max _{0 \leq k \leq\left\lfloor t / \mu^{2}\right\rfloor}\left(\sigma B\left(\mu^{2} k\right)-\mu^{2} k\right) \rightarrow \max _{0 \leq s \leq t}(\sigma B(s)-s) \quad \text { a.s. }
$$

as $\mu \nearrow 0$, and

$$
\begin{aligned}
& \mathrm{E}\left[\prod_{k=0}^{\left\lfloor t / \mu^{2}\right\rfloor}\left(1-\bar{F}_{\xi}\left(\left(x-\sigma B\left(\mu^{2} k\right)+\mu^{2} k\right)|\mu|^{-2 / \alpha}\right)\right)\right. \\
& \left.\quad \times \mathbf{1}\left(x-|\mu|^{1 / 2 \alpha} \leq \max _{0 \leq k \leq\left\lfloor t / \mu^{2}\right\rfloor}\left(\sigma B\left(\mu^{2} k\right)-\mu^{2} k\right) \leq x-b|\mu|^{2 / \alpha}\right)\right] \\
& \quad \leq \mathrm{P}\left(x-|\mu|^{1 / 2 \alpha} \leq \max _{0 \leq k \leq\left\lfloor t / \mu^{2}\right\rfloor}\left(\sigma B\left(\mu^{2} k\right)-\mu^{2} k\right) \leq x-b|\mu|^{2 / \alpha}\right) \\
& \quad \rightarrow 0
\end{aligned}
$$

as $\mu \nearrow 0$. Furthermore, because $\max _{0 \leq s \leq t}(\sigma B(s)-s)$ is a random variable possessing a density, it follows that, conditional on the maximum of $\sigma B(s)-s$ being less than or equal to $x$, the maximum is almost surely strictly smaller than $x$. On a sample path on which the maximum is strictly less than $x-|\mu|^{1 / 2 \alpha}$, we have

$$
\begin{aligned}
& \prod_{k=0}^{\left\lfloor t / \mu^{2}\right\rfloor}\left(1-\bar{F}_{\xi}\left(\left(x-\sigma B\left(\mu^{2} k\right)+\mu^{2} k\right)|\mu|^{-2 / \alpha}\right)\right) \\
& \quad=\exp \left(\sum_{k=0}^{\left\lfloor t / \mu^{2}\right\rfloor} \log \left(1-\bar{F}_{\xi}\left(\left(x-\sigma B\left(\mu^{2} k\right)+\mu^{2} k\right)|\mu|^{-2 / \alpha}\right)\right)\right) \\
& \quad=\exp \left(\sum_{k=0}^{\left\lfloor t / \mu^{2}\right\rfloor} \log \left(1-b^{\alpha}\left(x-\sigma B\left(\mu^{2} k\right)+\mu^{2} k\right)^{-\alpha} \mu^{2}\right)\right)
\end{aligned}
$$


for $\mu$ sufficiently small that the maximum is smaller than $x-2|\mu|^{1 / 2 \alpha}$. Furthermore, for such values of $\mu$,

$$
\left(x-\sigma B\left(\mu^{2} k\right)+\mu^{2} k\right)^{-\alpha} \mu^{2} \leq 2|\mu|^{3 / 2}
$$

uniformly in $k$. Consequently, for such $\mu$,

$$
\begin{aligned}
\log (1 & \left.-b^{\alpha}\left(x-\sigma B\left(\mu^{2} k\right)+\mu^{2} k\right)^{-\alpha} \mu^{2}\right) \\
& =-b^{\alpha}\left(x-\sigma B\left(\mu^{2} k\right)+\mu^{2} k\right)^{-\alpha} \mu^{2}+O\left(\mu^{3}\right)
\end{aligned}
$$

uniformly in $k$, so that

$$
\begin{aligned}
& \sum_{k=0}^{\left\lfloor t / \mu^{2}\right\rfloor} \log \left(1-\bar{F}_{\xi}\left(\left(x-\sigma B\left(\mu^{2} k\right)+\mu^{2} k\right)|\mu|^{-2 / \alpha}\right)\right) \\
& \quad=-b^{\alpha} \sum_{k=0}^{\left\lfloor t / \mu^{2}\right\rfloor}\left(x-\sigma B\left(\mu^{2} k\right)+\mu^{2} k\right)^{-\alpha} \mu^{2}+o(1) \text { a.s. }
\end{aligned}
$$

as $\mu \nearrow 0$. On paths on which the maximum is strictly less than $x-2|\mu|^{1 / 2 \alpha}$,

$$
(x-\sigma B(s)+\mu s)^{-\alpha}
$$

is continuous and bounded in $s$ over $[0, t]$. It follows that, because the above sum is a Riemann approximation to the integral, we have

$$
\sum_{j=0}^{\left\lfloor t / \mu^{2}\right\rfloor}\left(x-\sigma B\left(\mu^{2} k\right)+\mu^{2} k\right)^{-\alpha} \mu^{2} \rightarrow \int_{0}^{t}(x-\sigma B(s)+s)^{-\alpha} \mathrm{d} s
$$

as $\mu \nearrow 0$ for such paths. In view of (15)-(17), we therefore may conclude that

$$
\begin{array}{r}
\prod_{k=0}^{\left\lfloor t / \mu^{2}\right\rfloor}\left(1-\bar{F}_{\xi}\left(\left(x-\sigma B\left(\mu^{2} k\right)+\mu^{2} k\right)|\mu|^{-2 / \alpha}\right)\right) \\
\quad \rightarrow \exp \left(-b^{\alpha} \int_{0}^{t}(x-\sigma B(s)+s)^{-\alpha} \mathrm{d} s\right)
\end{array}
$$

for such paths. However, the left-hand side of (18) is a family of random variables that is bounded in $\mu$ (by the constant 1), so the bounded convergence theorem, together with (12), (13), and (14), then yields the desired conclusion.

Theorem 2 suggests an approximation for the maximum of a perturbed random walk that is appropriate when the perturbations have a right tail that is Pareto. In particular, for a given perturbed random walk $\left(S_{j}+\xi_{j}: j \geq 0\right.$ ) with Pareto perturbations, the approximation suggested by Theorem 2 is

$$
\begin{aligned}
\mathrm{P}\left(\max _{0 \leq k \leq n}\left(S_{k}+\xi_{k}\right)>x\right) \stackrel{\mathrm{D}}{\approx} 1-\mathrm{E}[ & \exp \left(-b^{\alpha} \int_{0}^{n}(x-\sigma B(s)-\mu s)^{-\alpha} \mathrm{d} s\right) \\
& \left.\times \mathbf{1}\left(\max _{0 \leq s \leq n}(\sigma B(s)+\mu s) \leq x-b\right)\right] .
\end{aligned}
$$


The parameters $\mu$ and $\sigma^{2}$ appearing in (19) are defined as in (10) and (11), while the quantities $b$ and $\alpha$ are the parameters that define the Pareto distribution of the perturbations. As for Theorem 1, we expect the above approximation to be good over spatial scales of order $1 /|\mu|$ when $|\mu|$ is small, the time $n$ is of order $1 / \mu^{2}$, and the perturbations are of order $|\mu|^{(2-\alpha) / \alpha}$.

\section{Infinite-horizon limit theory with light tails}

In the previous two sections, we used Theorems 1 and 2 to discuss the heavy-traffic setting, providing approximations to the maximum of perturbed random walks over finite time intervals. However, in view of the discussion in Section 2 of Araman and Glynn (2004), perhaps the most interesting characteristic of the perturbed random walk is the distribution of the alltime maximum $M_{\infty}$. Since $M_{\infty}$ involves the 'infinite-time' behavior of the perturbed random walk, we cannot conclude from Theorems 1 and 2 that our Brownian approximations are appropriate for use as approximations of $M_{\infty}$. In the remainder of this section, we provide rigorous justification for such Brownian approximations to the distribution of $M_{\infty}$. We start by considering light-tailed perturbations by (slightly) strengthening Assumption 2.

Assumption 4. Suppose that $\left(\xi_{j}: j \geq 0\right)$ is a stationary sequence for which there exist positive constants $\gamma$ and $\beta$ such that

$$
\frac{\max _{0 \leq k \leq n} \xi_{k}}{(\log n)^{\gamma}} \rightarrow \beta \text { a.s. }
$$

as $n \rightarrow \infty$.

See Zeevi and Glynn (1999) for such almost-sure convergence results in the stationary sequence setting.

Clearly, $M_{\infty}$ is finite valued only when the underlying random walk has negative drift. We therefore restrict attention, in the following results, to the case in which $\mu \nearrow 0$.

Theorem 3. Assume that Assumption 1 and Assumption 4 hold, and suppose that $\kappa(\mu) \sim$ $c\left(|\mu|(\log (1 /|\mu|))^{\gamma}\right)^{-1}$ as $\mu \nearrow 0$, where $c$ is a positive constant. Then

$$
|\mu| M_{\infty}(\mu) \stackrel{\mathrm{D}}{\rightarrow} \max _{t \geq 0}(\sigma B(t)-t)+2^{\gamma} \beta c
$$

as $\mu \nearrow 0$.

Proof. Fix an $\varepsilon>0$. We first set $t^{*}=\max \left(1, t_{1}, t_{2}\right)$, where $t_{1}$ and $t_{2}$ are chosen so that

$$
\mathrm{P}\left(\max _{t \geq t_{1}}(\sigma B(t)-t) \geq-\left|\xi_{0}\right|\right)<\varepsilon
$$

and

$$
\frac{(\log t+2)^{\gamma}}{t} \leq \frac{1}{16 \beta c} \quad \text { for } t \geq t_{2}
$$

Observe that

$$
\begin{aligned}
& \left|\mathrm{P}\left(\max _{0 \leq t \leq t^{*}}(\sigma B(t)-t)+2^{\gamma} \beta c \in \cdot\right)-\mathrm{P}\left(\max _{t \geq 0}(\sigma B(t)-t)+2^{\gamma} \beta c \in \cdot\right)\right| \\
& \quad \leq \mathrm{P}\left(\max _{t \geq t^{*}}(\sigma B(t)-t) \geq 0\right)<\varepsilon .
\end{aligned}
$$

Assumption 1 guarantees that there exists a finite-valued random variable $L_{1}$ such that

$$
\left|S_{k}-\sigma B(k)\right| \leq \frac{1}{4} k^{1 / 2}
$$


for $k \geq L_{1}$, whereas Assumption 4 ensures the existence of an $L_{2}$ for which

$$
\max _{0 \leq k \leq n} \xi_{k} \leq 2 \beta(\log n)^{\gamma}
$$

for $n \geq L_{2}$. Set $L=\max \left(L_{1}, L_{2}\right)$ and note that

$$
\begin{aligned}
&\left|\mathrm{P}\left(|\mu| M_{\left\lfloor t^{*} / \mu^{2}\right\rfloor}(\mu) \in \cdot\right)-\mathrm{P}\left(|\mu| M_{\infty}(\mu) \in \cdot\right)\right| \\
& \leq \mathrm{P}\left(\max _{k \geq\left\lceil t^{*} / \mu^{2}\right\rceil}\left(|\mu| S_{k}(\mu)+|\mu| \xi_{k}(\mu)\right) \geq|\mu| \xi_{0}(\mu)\right) \\
& \leq \mathrm{P}\left(\max _{k \geq\left\lceil t^{*} / \mu^{2}\right\rceil}\left(|\mu| S_{k}(\mu)+|\mu| \xi_{k}(\mu)\right) \geq|\mu| \xi_{0}(\mu), L \leq\left\lceil t^{*} / \mu^{2}\right\rceil\right) \\
&+\mathrm{P}\left(L>\left\lceil t^{*} / \mu^{2}\right\rceil\right) .
\end{aligned}
$$

On $\left\{L \leq\left\lceil t^{*} / \mu^{2}\right\rceil\right\}$, we have

$$
\begin{aligned}
|\mu| \xi_{k}(\mu) & \leq \frac{2 c}{(\log 1 /|\mu|)^{\gamma}} \max _{0 \leq j \leq k} \xi_{j} \\
& \leq 4 \beta c \frac{(\log k)^{\gamma}}{(\log 1 /|\mu|)^{\gamma}}
\end{aligned}
$$

for $k \geq\left\lceil t^{*} / \mu^{2}\right\rceil$ and $\mu$ sufficiently small. However, $(\log k)^{\gamma} / k$ decreases for large $k$, meaning that, for $|\mu| \leq 1 / \mathrm{e}$ and $k \geq\left\lceil t^{*} / \mu^{2}\right\rceil$, we have

$$
\begin{aligned}
4 \beta c \frac{(\log k)^{\gamma}}{(\log 1 /|\mu|)^{\gamma}} & \leq 4 \beta c \frac{\left(\log t^{*} / \mu^{2}\right)^{\gamma} k}{t^{*} / \mu^{2}(\log 1 /|\mu|)^{\gamma}} \\
& =4 \beta c\left(\frac{\log t^{*}}{\log 1 /|\mu|}+2\right)^{\gamma} \frac{1}{t^{*}} k \mu^{2} \\
& \leq 4 \beta c\left(\log t^{*}+2\right)^{\gamma} \frac{1}{t^{*}} k \mu^{2} \\
& \leq \frac{1}{4} k \mu^{2}
\end{aligned}
$$

where the definition of $t_{2}$ was used in the final inequality. On $\left\{L \leq\left\lceil t^{*} / \mu^{2}\right\rceil\right\}$, we also have

$$
|\mu|\left|S_{k}-\sigma B(k)\right| \leq|\mu| \frac{1}{4} k^{1 / 2} \leq|\mu| \frac{1}{4} k^{1 / 2} k^{1 / 2}|\mu| \leq \frac{1}{4} k \mu^{2}
$$

for $k \geq\left\lceil t^{*} / \mu^{2}\right\rceil$, where the fact that $t^{*} \geq 1$ was used in the second inequality above.

Combining (22)-(24), we conclude that, on $\left\{L \leq\left\lceil t^{*} / \mu^{2}\right\rceil\right\}$, we have

$$
|\mu| S_{k}+|\mu| \xi_{k}(\mu) \leq|\mu| \sigma B(k)-\frac{1}{2} \mu^{2} k
$$

for $k \geq\left\lceil t^{*} / \mu^{2}\right\rceil$. Hence, for $\mu$ sufficiently small,

$$
\begin{aligned}
\mathrm{P}\left(\max _{k \geq\left\lceil t^{*} / \mu^{2}\right\rceil}\left(|\mu| S_{k}+|\mu| \xi_{k}(\mu)\right) \geq|\mu| \xi_{0}(\mu)\right) \\
\quad \leq \mathrm{P}\left(\max _{k \geq\left\lceil t^{*} / \mu^{2}\right\rceil}\left(|\mu| \sigma B(k)-\mu^{2} k\right) \geq|\mu| \xi_{0}(\mu)\right) \\
\quad \leq \mathrm{P}\left(\max _{s \geq t^{*}}\left(\sigma B(s)-\frac{1}{2} s\right) \geq-\left|\xi_{0}\right|\right) \\
\quad<\varepsilon .
\end{aligned}
$$


Inequalities (20), (21), and (25) imply that

$$
\begin{aligned}
\mid \mathrm{P}(|\mu| & \left.M_{\infty}(\mu) \in \cdot\right)-\mathrm{P}\left(\max _{t \geq 0}(\sigma B(t)-t)+2^{\gamma} \beta c \in \cdot\right) \mid \\
\leq & \left|\mathrm{P}\left(|\mu| M_{\infty}(\mu) \in \cdot\right)-\mathrm{P}\left(|\mu| M_{\left\lfloor t^{*} / \mu^{2}\right\rfloor}(\mu) \in \cdot\right)\right| \\
& +\left|\mathrm{P}\left(\max _{t \geq 0}(\sigma B(t)-t)+2^{\gamma} \beta c \in \cdot\right)-\mathrm{P}\left(\max _{0 \leq t \leq t *}(\sigma B(t)-t)+2^{\gamma} \beta c \in \cdot\right)\right| \\
\leq & 2 \varepsilon+\mathrm{P}\left(L>\left\lceil t^{*} / \mu^{2}\right\rceil\right) .
\end{aligned}
$$

By first letting $\mu \nearrow 0$ and then letting $\varepsilon \searrow 0$, we obtain the desired result.

Theorem 3 suggests an approximation for the distribution of $M_{\infty}$ that is appropriate to perturbed random walks with negative drift, for which the perturbations have a right tail of the type described in (1). However, in developing the approximation, care must be taken, since naive substitution of $n=\infty$ into the finite-time approximation (9) leads to a right-hand side that is infinite. The key is to take advantage of the 'diffusion scaling' that is implicit in all the results of this section. In particular, the results describe the spatial fluctuations of order $1 /|\mu|$ that occur over time scales of order $1 / \mu^{2}$. Thus, in order that the maximum of a perturbed random walk exceed a level of order $1 /|\mu|$, roughly $1 / \mu^{2}$ time units are required. This suggests that the implicit time scale that is relevant in approximating the all-time maximum $M_{\infty}$ is a time of order $1 / \mu^{2}$, meaning that the logarithmic term in (9) is then roughly given by $2^{\gamma}(\log 1 / \mu)^{\gamma}$. This yields the approximation

$$
M_{\infty} \stackrel{\mathrm{D}}{\approx} \max _{t \geq 0}(\sigma B(t)+\mu t)+2^{\gamma} \beta(\log 1 /|\mu|)^{\gamma}
$$

where $\mu, \sigma^{2}, \gamma$, and $\beta$ are defined as in (9). In view of Theorem 3, (26) should give reasonable approximations over spatial scales of order $1 /|\mu|$ when $\mu$ is small and negative and the perturbations are roughly of order $\kappa(|\mu|)$.

\section{Infinite-horizon limit theory with heavy tails}

Finally, we turn again to heavy-tailed perturbations that satisfy Assumption 3, to study the infinite-horizon analog of (19). We note that the term $(x-\sigma B(s)-\mu s)^{-\alpha}$ appearing there is almost surely asymptotic to $(-\mu s)^{-\alpha}$ as $s \rightarrow \infty$. Hence, even when the drift is negative, it follows that

$$
\int_{0}^{\infty}(x-\sigma B(s)-\mu s)^{-\alpha} \mathrm{d} s=\infty \quad \text { a.s. }
$$

for $0<\alpha \leq 1$, in which case the right-hand side of (19) describes an improper random variable. Consequently, the approximation described by Theorem 3 has a finite all-time maximum only when the perturbations follow a Pareto distribution with finite mean. This is in fact consistent with Proposition 2 of Araman and Glynn (2004). The latter result states that a necessary and sufficient condition for the all-time maximum of a perturbed random walk to be finite almost surely is that the perturbation sequence (assumed to consist of independent, identically distributed, and nonnegative random variables) admits a finite mean. Therefore, we henceforth restrict our attention to the case in which $\alpha>1$. It is then easily seen that the right-hand side of (19) defines a proper (finite-valued) random variable. 
Theorem 4. Assume that Assumption 1 and Assumption 3 hold, with $\alpha>1$. If $\kappa(\mu)=$ $|\mu|^{(2-\alpha) / \alpha}$ then

$$
\begin{aligned}
\mathrm{P}(|\mu| & \left.M_{\infty}(\mu)>x\right) \\
& \rightarrow 1-\mathrm{E}\left[\exp \left(-b^{\alpha} \int_{0}^{\infty}(x-\sigma B(s)+s)^{-\alpha} \mathrm{d} s\right) \mathbf{1}\left(\max _{t \geq 0}(\sigma B(t)-t) \leq x\right)\right]
\end{aligned}
$$

as $\mu \nearrow 0$.

The proof of Theorem 4 follows an argument virtually identical to that of Theorem 3 , and is therefore omitted.

The infinite-horizon approximation suggested by Theorem 4 is

$$
\begin{aligned}
\mathrm{P}\left(\max _{k \geq 0}\left(S_{k}+\xi_{k}\right)>x\right) & \\
\approx 1-\mathrm{E}[ & \exp \left(-b^{\alpha} \int_{0}^{\infty}(x-\sigma B(s)-\mu s)^{-\alpha} \mathrm{d} s\right) \\
& \left.\times \mathbf{1}\left(\max _{t \geq 0}(\sigma B(t)+\mu t) \leq x-b\right)\right],
\end{aligned}
$$

where $\mu, \sigma^{2}, \alpha$, and $b$ are defined as in (19). We expect (27) to give reasonable approximations over spatial scales of order $1 /|\mu|$ when $\mu$ is small and negative and the perturbations are roughly of order $|\mu|^{(2-\alpha) / \alpha}$.

The remainder of this section is devoted to a discussion of how to compute the expectation appearing in (27). We start by noting that, since

$$
\begin{aligned}
w(y) & :=\mathrm{P}\left(\max _{t \geq 0}(\sigma B(t)+\mu t) \leq-y-b\right) \\
& =1-\exp \left(-\frac{2 \mu(y+b)}{\sigma^{2}}\right)
\end{aligned}
$$

is known in closed form for $y \leq-b$ (and $\mu$ negative), the key is to compute

$$
\mathrm{E}\left[\exp \left(-b^{\alpha} \int_{0}^{\infty}|W(s)|^{-\alpha} \mathrm{d} s\right) \mid W(0)=-x, \max _{t \geq 0} W(t) \leq-b\right],
$$

where $W=(W(t): t \geq 0)$ is Brownian motion with drift $\mu$ and variance parameter $\sigma^{2}$. The process $W$, conditioned on $\max \{W(t): t \geq 0\} \leq-b$, is itself a diffusion. To (nonrigorously) compute the generator of the conditioned process $Y$, let

$$
\tilde{A}=\mu \frac{\mathrm{d}}{\mathrm{d} x}+\frac{\sigma^{2}}{2} \frac{\mathrm{d}^{2}}{\mathrm{~d} x^{2}}
$$

be the generator of Brownian motion with drift $\mu$ and variance parameter $\sigma^{2}$, and observe that, for $y<-b$ and any function $f$ with minimal measurability conditions, we have

$$
\begin{aligned}
\mathrm{E}[f(Y(h)) \mid Y(0)=y] \\
\quad=\mathrm{E}\left[f(W(h)) \mid W(0)=y, \max _{t \geq 0} W(t) \leq-b\right] \\
\quad \approx \frac{1}{w(y)} \mathrm{E}[f(W(h)) w(W(h)) \mid W(0)=y] \\
\quad=\frac{1}{w(y)}[f(y) w(y)+(\tilde{A} f w)(y) h+o(h)] .
\end{aligned}
$$


This suggests that the generator $A$ of the conditioned process $Y$ is given by

$$
\begin{aligned}
(A f)(y) & =\mu \frac{\mathrm{d}}{\mathrm{d} y}(f(y) w(y))+\frac{\sigma^{2}}{2} \frac{\mathrm{d}^{2}}{\mathrm{~d} y^{2}}(f(y) w(y)) \\
& =(\tilde{A} f)(y)+\sigma^{2} \frac{w^{\prime}(y)}{w(y)} f^{\prime}(y) \\
& =\left[\mu+\frac{2 \mu}{\exp \left(2 \mu(y+b) / \sigma^{2}\right)-1}\right] f^{\prime}(y)+\frac{\sigma^{2}}{2} f^{\prime \prime}(y)
\end{aligned}
$$

for $y<-b$ and $f$ twice continuously differentiable; a related (nonrigorous) computation can be found on pp. 271-272 of Karlin and Taylor (1981). Note that the drift decreases to $-\infty$ as $y$ approaches $-b$, making $-b$ an inaccessible boundary for $Y$ (as expected). To make the above computation rigorous, a 'change-of-measure' argument, based on Girsanov's formula, can be applied, as in Glynn and Thorisson (2001).

Given the above calculation, to compute (28) we now must find $v(-x)$, given by

$$
v(y)=\mathrm{E}\left[\exp \left(-b^{\alpha} \int_{0}^{\infty}|Y(t)|^{-\alpha} \mathrm{d} t\right) \mid Y(0)=y\right] .
$$

The function $v(\cdot)$ can be found by solving an appropriate differential equation.

Proposition 1. Suppose that there exists a twice-continuously differentiable, positive, decreasing function $v$ defined on $[-\infty,-b)$, with $v(-\infty)=1$, satisfying

$$
\left[\mu+\frac{2 \mu}{\exp \left(2 \mu(y+b) / \sigma^{2}\right)-1}\right] v^{\prime}(y)+\frac{\sigma^{2}}{2} v^{\prime \prime}(y)-b^{\alpha}|y|^{-\alpha} v(y)=0 \quad(y<-b) .
$$

Then

$$
v(y)=\mathrm{E}\left[\exp \left(-b^{\alpha} \int_{0}^{\infty}|Y(t)|^{-\alpha} \mathrm{d} t\right) \mid Y(0)=y\right] \quad(y<-b) .
$$

Proof. The process $Y$ satisfies the stochastic differential equation

$$
\mathrm{d} Y(t)=\left[\mu+\frac{2 \mu}{\exp \left(2 \mu(Y(t)+b) / \sigma^{2}\right)-1}\right] \mathrm{d} t+\frac{\sigma^{2}}{2} \mathrm{~d} B(t) .
$$

Itô's formula then ensures that

$$
\begin{aligned}
\mathrm{d}(\exp ( & \left.\left.-b^{\alpha} \int_{0}^{t}|Y(s)|^{-\alpha} \mathrm{d} s\right) v(Y(t))\right) \\
= & \exp \left(-b^{\alpha} \int_{0}^{t}|Y(s)|^{-\alpha} \mathrm{d} s\right)\left((A v)(Y(t))-b^{\alpha}|Y(t)|^{-\alpha} v(Y(t))\right) \mathrm{d} t \\
& +\exp \left(-b^{\alpha} \int_{0}^{t}|Y(s)|^{-\alpha} \mathrm{d} s\right) v^{\prime}(Y(t)) \sigma \mathrm{d} B(t) \\
= & \exp \left(-b^{\alpha} \int_{0}^{t}|Y(s)|^{-\alpha} \mathrm{d} s\right) v^{\prime}(Y(t)) \sigma \mathrm{d} B(t),
\end{aligned}
$$

where the second equality relies on the fact that $v$ satisfies the stated differential equation. If

$$
T_{n}=\inf \left\{t \geq 0: Y(t) \leq-n \text { or } Y(t) \geq-b-\frac{1}{n}\right\}
$$


the boundedness of $v^{\prime}$ on $[-n,-b-1 / n]$ implies that the optional sampling theorem may be applied at time $T_{n}$ to the stochastic integral, yielding the equality

$$
v(y)=\mathrm{E}\left[\exp \left(-b^{\alpha} \int_{0}^{T_{n}}|Y(s)|^{-\alpha} \mathrm{d} s\right) v\left(Y\left(T_{n}\right)\right) \mid Y(0)=y\right] .
$$

Since $W(t) \rightarrow-\infty$ a.s. and the conditioning event $\{\max \{W(t): t \geq 0\} \leq-b\}$ has positive probability, it follows that $Y(t) \rightarrow-\infty$ a.s. as $t \rightarrow \infty$. The path continuity of $Y$ therefore permits us to conclude that $Y\left(T_{n}\right) \rightarrow-\infty$ a.s., meaning that $v\left(Y\left(T_{n}\right)\right) \rightarrow 1$. The positive and decreasing nature of $v$ implies that $v\left(Y\left(T_{n}\right)\right)$ is bounded in absolute value by 1 . Thus, the bounded convergence theorem applied to (29) yields the desired conclusion.

The authors are unaware of any closed-form solution to the differential equation. Consequently, it must be solved numerically. An alternative is to develop asymptotics for the solution.

Theorem 5. Suppose that $\mu<0$ and $\alpha>2$. Then

$$
\begin{aligned}
1- & \operatorname{Eexp}\left(-b^{\alpha} \int_{0}^{\infty}(x-\sigma B(s)-\mu s)^{-\alpha} \mathrm{d} s\right) \mathbf{1}\left(\max _{t \geq 0}(\sigma B(t)+\mu t) \leq x-b\right) \\
= & \frac{b^{\alpha}}{|\mu| \alpha} x^{1-\alpha}+b^{\alpha} \frac{\sigma^{2}}{2 \mu^{2}} x^{-\alpha}+o\left(x^{-\alpha}\right)
\end{aligned}
$$

as $x \rightarrow \infty$.

Proof. The scaling properties of Brownian motion imply that

$$
\begin{aligned}
& E \exp \left(-b^{\alpha} \int_{0}^{\infty}(x-\sigma B(s)-\mu s)^{-\alpha} \mathrm{d} s\right) \mathbf{1}\left(\max _{t \geq 0}(\sigma B(t)+\mu t) \leq x-b\right) \\
& \quad=\mathrm{E} \exp \left(-b^{\alpha} x^{1-\alpha} J(x)\right) I(x),
\end{aligned}
$$

where

$$
\begin{aligned}
& J(x)=\int_{0}^{\infty}\left(1-\sigma x^{-1 / 2} B(r)-\mu r\right)^{-\alpha} \mathrm{d} r \\
& I(x)=\mathbf{1}\left(\max _{r \geq 0}\left(\sigma x^{-1 / 2} B(r)+\mu r\right) \leq 1-\frac{b}{x}\right) .
\end{aligned}
$$

On $\{I(x)=1\}$, we can express the integrand of $J(x)$ as

$$
\begin{aligned}
\left(1-\sigma x^{-1 / 2} B(r)-\mu r\right)^{-\alpha}= & (1-\mu r)^{-\alpha}+\alpha(1-\mu r)^{-\alpha-1} \sigma B(r) x^{-1 / 2} \\
& +\frac{1}{2} \alpha(\alpha+1)(1-\mu r-\zeta(r, x))^{-\alpha-2} \sigma^{2} B^{2}(r) x^{-1},
\end{aligned}
$$

where $\zeta$ is some function of $r$ and $x$ such that $|\zeta(r, x)| \leq \sigma x^{-1 / 2}|B(r)|$. In fact,

$$
\begin{aligned}
(1-\mu r-\xi(r, x)) & \geq(1-\mu r) \wedge\left((1-\mu r)-x^{-1 / 2} \sigma B(r)\right) \\
& \geq(1-\mu r) \wedge\left(1-\mu r\left(1-x^{-1 / 2}\right)-x^{-1 / 2} R\right) \\
& =\left(1-\mu r\left(1-x^{-1 / 2}\right)-x^{-1 / 2} R\right),
\end{aligned}
$$


where $R=\max \{\sigma B(r)+\mu r: r \geq 0\}$. It follows that if $L=\sup \left\{t \geq 0:|\sigma B(t)|>\frac{1}{2}|\mu| t\right\}$ then, for $x \geq 1$, we have

$$
\begin{aligned}
x \mid J(x) & -\int_{0}^{\infty}(1-\mu r)^{-\alpha} \mathrm{d} r-\sigma x^{-1 / 2} \alpha \int_{0}^{\infty}(1-\mu r)^{-\alpha-1} B(r) \mathrm{d} r \mid I(x) \\
\leq & \frac{\alpha(\alpha+1)}{2} \sigma^{2} \int_{0}^{\infty}(1-\mu r-\zeta(r, x))^{-\alpha-2} B^{2}(r) \mathrm{d} r I(x) \mathbf{1}\left(R>\frac{x^{1 / 2}}{2}\right) \\
& +\frac{\alpha(\alpha+1)}{2} \sigma^{2} \int_{0}^{\infty}\left(\frac{1}{2}-\mu r\right)^{-\alpha-2} B^{2}(r) \mathrm{d} r I(x) \mathbf{1}\left(R \leq \frac{x^{1 / 2}}{2}\right) \\
\leq & \frac{\alpha(\alpha+1)}{2} \sigma^{2} \int_{0}^{L}(1-\mu r-\zeta(r, x))^{-\alpha-2} B^{2}(r) \mathrm{d} r I(x) \mathbf{1}\left(R>\frac{x^{1 / 2}}{2}\right) \\
& +\frac{\alpha(\alpha+1)}{2} \sigma^{2} \int_{L}^{\infty}\left(1-\frac{\mu r}{2}\right)^{-\alpha-2} B^{2}(r) \mathrm{d} r \\
& +\frac{\alpha(\alpha+1)}{2} \sigma^{2} \int_{0}^{\infty}\left(\frac{1}{2}-\mu r\right)^{-\alpha-2} B^{2}(r) \mathrm{d} r \\
\leq & \frac{\alpha(\alpha+1)}{2} \sigma^{2} 2^{\alpha+2} b^{-\alpha-2} R^{\alpha+2} \int_{0}^{L} B^{2}(r) \mathrm{d} r \\
& +\alpha(\alpha+1) \sigma^{2} \int_{0}^{\infty}\left(\frac{1}{2}-\frac{\mu r}{2}\right)^{-\alpha-2} B^{2}(r) \mathrm{d} r .
\end{aligned}
$$

Here, we have used the fact that $\left(1-\mu r-\sigma x^{-1 / 2} B(r)\right)^{-\alpha-2} \leq(x / b)^{\alpha+2}$ when $I(x)=1$ to obtain the third inequality above.

Recall that, for $w \geq 0$, we have

$$
1-w \leq \mathrm{e}^{-w} \leq 1-w+\frac{1}{2} w^{2} .
$$

Since $J(x) \geq 0$ on $\{I(x)=1\}$, we have

$$
x^{\alpha}\left|1-\exp \left(-b^{\alpha} x^{1-\alpha} J(x)\right)-b^{\alpha} x^{1-\alpha} J(x)\right| \leq \frac{1}{2} b^{2 \alpha} x^{2-\alpha} J^{2}(x) .
$$

Because $L$ and $R$ have exponential tails (for $L$ see Robbins et al. (1968), and for $R$ see Harrison (1985)), evidently $R^{\alpha+2} \int_{0}^{\infty} B^{2}(r) \mathbf{1}(L>r) \mathrm{d} r$ is integrable. The dominated convergence theorem, together with (30) and (31), shows that

$$
x^{\alpha}\left(1-\mathrm{E} \exp \left(-b^{\alpha} x^{1-\alpha} J(x)\right) I(x)\right)=b^{\alpha} x \mathrm{E} J(x) I(x)+o(1)
$$

as $x \rightarrow \infty$.

A path-by-path application of the dominated convergence theorem, taking advantage of the bound (30), yields

$$
\begin{aligned}
x J(x) I(x)= & x \int_{0}^{\infty}(1-\mu r)^{-\alpha} \mathrm{d} r+\sigma x^{-1 / 2} \alpha \int_{0}^{\infty}(1-\mu r)^{-\alpha-1} B(r) \mathrm{d} r \\
& +\frac{\alpha(\alpha+1)}{2} \sigma^{2} \int_{0}^{\infty}(1-\mu r)^{-\alpha-2} B^{2}(r) \mathrm{d} r+o(1) \quad \text { a.s. }
\end{aligned}
$$

as $x \rightarrow \infty$. The integrability of $R^{\alpha+2} \int_{0}^{L} B^{2}(r) \mathrm{d} r$ allows us to again apply the dominated convergence theorem, this time to pass expectations through (32). This proves the theorem. 
TABLE 1: Estimates of $\mathrm{P}\left(M_{\infty}>u\right)$ with exponentially distributed perturbations.

\begin{tabular}{lccccc}
\hline \multicolumn{1}{c}{$u$} & $\mathrm{P}\left(M_{\infty}>u\right)$ & Approximation & Relative error $(\%)$ & $L$ & $R$ \\
\hline 0.125 & 0.980 & 0.9997 & 2.1 & 0.980 & 0.980 \\
0.25 & 0.980 & 0.9997 & 2.0 & 0.979 & 0.980 \\
0.5 & 0.979 & 0.9996 & 2.1 & 0.979 & 0.980 \\
2.5 & 0.979 & 0.999 & 2.0 & 0.979 & 0.980 \\
5 & 0.979 & 0.999 & 2.0 & 0.978 & 0.979 \\
50 & 0.970 & 0.990 & 2.1 & 0.969 & 0.970 \\
500 & 0.885 & 0.903 & 2.0 & 0.884 & 0.885 \\
\hline
\end{tabular}

TABLE 2: Estimates of $\mathrm{P}\left(M_{\infty}>u\right)$ with Pareto-distributed perturbations.

\begin{tabular}{rccccc}
\hline \multicolumn{1}{c}{$u$} & $\mathrm{P}\left(M_{\infty}>u\right)$ & Approximation & Relative error $(\%)$ & $L$ & $R$ \\
\hline 2 & 0.9491 & 0.9992 & 5.3 & 0.9480 & 0.9503 \\
100 & 0.9021 & 0.9489 & 5.2 & 0.9011 & 0.9032 \\
400 & 0.7698 & 0.8101 & 5.2 & 0.7689 & 0.7707 \\
1000 & 0.5613 & 0.5904 & 5.2 & 0.5606 & 0.5620 \\
2000 & 0.3308 & 0.3482 & 5.3 & 0.3304 & 0.3312 \\
4000 & 0.1158 & 0.1213 & 4.7 & 0.1157 & 0.1159 \\
\hline
\end{tabular}

\section{Numerical results}

In this section, we present some experimental results to accompany our theoretical limiting results obtained above. We restrict the analysis to the infinite-horizon case. In particular, we compare the approximations suggested for the tail distribution of the all-time maximum of the perturbed random walk, through (26) and (27), to the values obtained through simulation. We respectively consider a light-tailed and a Pareto-tailed perturbation. For both cases, we assume the original random walk to be the difference of two exponentially distributed random variables, as in the $\mathrm{M} / \mathrm{M} / 1$ setting (such that $\mathrm{E} X_{1}=\mu<0$ ).

So, for the light-tailed perturbation we consider an exponentially distributed random variable. This clearly satisfies Assumption 3 with $\gamma=\beta=1$. For the simulation, we take $\mu=2$. Having Theorem 3 in mind, we let $\mathrm{E} \xi_{1}=(|\mu| \log (1 /|\mu|))^{-1}$. The results are based on 10000 replications and are reported in Table 1 . The last two columns represent the $95 \%$ confidence interval around the simulated value. We observe that the relative error is almost constant, independent of the values of $u$. It will improve as the mean random walk comes closer to 0 .

For the heavy-tailed perturbation, we take a Pareto perturbation as defined in Assumption 3, with $\alpha=3$ and $b=\frac{2}{3}$. We let $\mu=5$ and, to use Theorem 4 , we set $\mathrm{E} \xi=|\mu|^{2 / 3}$. The results are based on 5000 replications and are reported in Table 2. The relative error is around 5\%, independent of the threshold $u$, and will become smaller as $\mu$ comes closer to 0 .

\section{References}

Araman, V. F. And Glynn, P. W. (2004). Tail asymptotics for the maximum of a perturbed random walk. Submitted. CsÁKI, E. AND Csörgő, M. (1995). On additive functionals of Markov chains. J. Theoret. Prob. 8, 905-919.

Csörgő, M. AND Révész, P. (1981). Strong Approximations in Probability and Statistics. Academic Press, New York. Gerber, H. U. (1970). An extension of the renewal theorem and its application in the collective theory of risk. Skand. Aktuar. 1970, 205-210. 
Glasserman, P. AND LiU, T. W. (1997). Corrected diffusion approximations for a multistage production-inventory system. Math. Operat. Res. 22, 186-201.

GlynN, P. W. (1998). Strong approximations in queueing theory. In Asymptotic Methods in Queueing Theory (Ottawa, ON, 1997), North-Holland, Amsterdam, pp. 135-150.

Glynn, P. W. and Thorisson, H. (2001). Two-sided taboo limits for Markov processes and associated perfect simulation. Stoch. Process. Appl. 91, 1-20.

GlynN, P. W. AND ZeEvi, A. J. (2000). Estimating tail probabilities in queues via external statistics. In Analysis of Communication Networks: Call Centres, Traffic and Performance (Toronto, ON, 1998; Fields Inst. Commun. 28), American Mathematical Society, Providence, RI, pp. 135-158.

Harrison, J. M. (1985). Brownian Motion and Stochastic Flow Systems. John Wiley, New York.

Karlin, S. And Taylor, H. M. (1981). A Second Course in Stochastic Processes. Academic Press, New York.

Philipp, W. And Stout, W. (1975). Almost sure invariance principles for partial sums of weakly dependent variables. Mem. Amer. Math. Soc. 1975, No. 161.

Robbins, H., Siegmund, D. And Wendel, J. (1968). The limiting distribution of the last time $s_{n} \geq$ ne. Proc. Nat. Acad. Sci. USA 61, 1228-1230.

SCHLEgEL, S. (1998). Ruin probabilities in perturbed risk models. Insurance Math. Econom. 22, 93-104.

Schmidl, H. (1995). Cramér-Lundberg approximations for ruin probabilities of risk processes perturbed by diffusion. Insurance Math. Econom. 16, 135-149.

Woodroofe, M. (1982). Nonlinear Renewal Theory in Sequential Analysis (CBMS-NSF Regional Conf. Ser. Appl. Math. 39). Society for Industrial and Applied Mathematics, Philadelphia, PA.

ZeEvi, A. J. AND GlynN, P. W. (1999). On the maximum workload in a queue fed by fractional Brownian motion. Ann. Appl. Prob. 10, 1084-1099. 